\title{
Optimal pollution control with distributed delays
}

\section{Emmanuelle AUGERAUD-VERON}

MIA

Université La Rochelle

\&

\section{Marc LEANDRI}

GREThA, CNRS, UMR 5113

Université de Bordeaux

$\&$

INRA LAMETA

\section{Cahiers du GREThA}

$$
\begin{gathered}
n^{\circ} \text { 2013-03 } \\
\text { January }
\end{gathered}
$$




\title{
Contrôle optimal de la pollution avec retards distribués
}

\section{Résumé}

Nous présentons un modèle de contrôle optimal de la pollution avec des retards distribués dans la dynamique d'accumulation du stock de pollution. Nous résolvons analytiquement le système dynamique complexe qui résulte de l'introduction de ces retards en utilisant des formes fonctionnelles générales ainsi qu'une structure de distribution qui couvre une large gamme de distributions. Notre contribution enrichit la littérature d'optimisation dynamique qui s'intéressait jusque là principalement au cas de simples retards discrets et développe une méthode originale pour traiter les problèmes de contrôle avec des équations différentielles mixtes. Nos résultats montrent l'impact qualitatif de ces retards sur les trajectoires optimales et identifient les conditions de stabilité et d'apparition de cycles limites.

Mots-clés : Contrôle optimal de la pollution, Retards Distribués, Equations différentielles mixtes, Bifurcation de Hopf

\section{Optimal pollution control with distributed delays}

\begin{abstract}
We present a model of optimal stock pollution control with distributed delays in the stock accumulation dynamics. Using generic functional forms and a distribution structure that covers a wide range of distributions, we solve analytically the complex dynamic system that arises from the introduction of these distributed delays. Our contribution extends the dynamic optimization literature that focused on the single discrete delay case and develops an original method to address control problems with mixed type functional differential equations. Our results show the qualitative impact of acknowledging these distributed delays on the optimal pollution paths dynamics and identify the occurrence of limit cycles and the stability conditions of such a model that can be used to design efficient environmental policies.
\end{abstract}

Keywords: Optimal Pollution Control, Distributed Delays, Mixed Type Functional Differential Equations, Hopf Bifurcation

JEL: C61, Q5

Reference to this paper: AUGERAUD-VERON Emmanuelle, LEANDRI Marc (2013), Optimal pollution control with distributed delays, Cahiers du GREThA, $\mathrm{n}^{\circ} 2013-03$.

http://ideas.repec.org/p/grt/wpegrt/2013-03.html. 


\section{Introduction}

Since the seminal contributions of Keeler et al. (1972), partial equilibrium stock pollution control models have been discussed and enriched in various ways with the introduction of uncertainty, multiple pollutants, irreversibility, technological change, etc. However, apart from a few exceptions presented below, this vast literature assumes systematically that the time of emission is tantamount to the time of contamination. This assumption leaves aside a crucial aspect of many pollution problems that feature significant delays in the accumulation process. For instance the contamination of aquifers by leaching nitrates from agricultural sources can occur several decades later (Bordenave et al., 1999), which explains in some cases why reductions in nitrogen-loaded inputs are not immediately followed by a decrease in downstream water pollution (Grimvall et al., 2000).

From a theoretical point of view, the addition of these delays to the standard optimal stock pollution control framework modifies the properties of the optimal pollution path. Winkler (2011) proves that a modified model with a discrete delay will display monotonic optimal paths if the objective function is separable in both stock and control variables, otherwise oscillatory paths may take place. Using such a separable objective function in a model with heterogeneous polluters, Bourgeois and Jayet (2011) show that higher time lags lead to a higher optimal pollution stock at the steady state and that this effect is amplified by asymmetric information. The common feature of these contributions is that they use a single discrete delay, assuming that an emission at time $t$ will reach entirely and systematically the pollution stock at time $t+\tau$. As we will show this kind of delay merely translates the dynamic path and leaves its mathematical properties relatively unaffected.

This model with a discrete delay implies nonetheless that the accumulation process is perfectly homogenous and it ignores the uncertainty that characterizes the accumulation velocity of polluting emissions. Site-specific conditions such as soil heterogeneity in the case of water contamination by nutriments or temperature and pressure variations in the case of greenhouse gases can cause significant variability in the time frame of pollutants accumulation. To better capture the intricate lags phenomena at stake, the time of accumulation of these pollutants should in fact be distributed along a time interval following the emission. Such distributed delays draw light on the challenging task of assessing the link between the time and amount of emissions and the time and intensity of the damage they trigger. They raise significant technical difficulties which, contrary the single discrete delay model, cannot be overcome easily even for a separable objective function. The application of Pontryagin's principle to this model with distributed delays gives rise to a system of optimal conditions that includes at the same time leads and lags, turning the system into Mixed Type Functional Differential Equations (MFDE). To our knowledge these delays have never been dealt with in optimal pollution control problems with general functions. Federico et al. (2010) do address a similar class of problems but they use specific

forms in order to solve explicitly the Hamilton Jacobi Bellman equations.

Our aim is to characterize analytically these complex dynamics and the 
stability conditions of the model using general functional forms. To do so we extend the approach used by Boucekkine et al. (2010) for vintage capital and we resort to an original method to address the MFDE at stake. Our results are based on a distribution structure that can cover a wide range of specific distributions. Our theoretical contribution finds significant applications in the design and calibration of economic environmental policies but could also be applied beyond this field to various other economic dynamic systems exhibiting similar delays (advertising, capital building,...).

After presenting in section 2 the general optimal stock pollution control model with delays and reassessing analytically the main properties of the single discrete delay problem, we introduce distributed delays in Section 3 under the most general form and we characterize the properties of the dynamic system with MFDE. Section 4 concludes.

\section{Introducing delays in the standard optimal pol- lution control model}

We consider the introduction of delays in a standard dynamic partial equilibrium model including a representative producer/polluter and the environmental damage undergone by society.

\subsection{The model}

The standard social planner problem is

$$
\max _{p(.)} \int_{0}^{\infty}[f(\theta p(t))-D(c(t))] e^{-\rho t} d t
$$

where $f(\theta p(t))$ is the private benefit derived from the polluting emissions $p(t), D(c(t))$ is the environmental damage caused by the pollution stock $c(t)$ and $\rho$ is the social discount rate with $\rho \in] 0,1[. f$ and $D$ feature the standard properties of the literature: $f$ positive, non-decreasing, concave, defined over $R^{+}$and respecting the Inada conditions and $D$ increasing, convex and such that $D(0)=0$. Assuming a standard linear relation between production intensity and polluting emission, we define $\theta$ as the constant technological pollution factor, $\theta>0$.

In order to study the influence of delays on the optimal control problem, we will consider a general expression of the pollution accumulation process that will allow us to address various types of delays. The standard accumulation equation can be written, with $\beta$ the natural decay rate of pollution

$$
\dot{c}(t)=-\beta c(t)+\frac{\int_{t-\tau_{2}}^{t-\tau_{1}} p(u) \mu(t-u) d u}{\int_{\tau_{1}}^{\tau_{2}} \mu(u) d u}
$$


where $0 \leq \tau_{1}<\tau_{2} \leq \infty$ and $\mu($.$) is a measure function on \left[\tau_{1}, \tau_{2}\right]$. This general writing under the form of a normalized measure is convenient to enclose a wide range of distribution structures. The choice of the measure $\mu($.$) will$ depend on the specific accumulation process and the normalization is necessary to guarantee that no extra pollution is created ex nihilo in the system.

Pollution emitted at time $t$ will be released in several weighted loads, distributed a priori across a time interval $\left[t+\tau_{1}, t+\tau_{2}\right)$. More precisely, pollution emitted at time $t$ will reach the stock at time $t+u$, with weight $\mu(t+u)$ where $u \in\left[\tau_{1}, \tau_{2}\right)$.

With this writing, the benchmark model corresponds to the single Dirac measure $\mu(v)=\delta_{0}(v)$, where the Dirac measure is defined, for any $a \in \mathbb{R}$ by

$$
\delta_{a}(v)= \begin{cases}1, & \text { if } v=a \\ 0, & \text { otherwise. }\end{cases}
$$

In this case the accumulation of pollution is instantaneous and the accumulation dynamics is written as

$$
\dot{c}(t)=-\beta c(t)+p(t)
$$

For an homogeneously delayed accumulation process, we use the single Dirac measure $\mu(v)=\delta_{\tau}(v)$, where the delay $\tau$ is strictly positive and $\tau \in\left[\tau_{1}, \tau_{2}\right)$. The accumulation rewrites as the classical linear discrete delay differential equation such as it can be found in Winkler (2011).

$$
\dot{c}(t)=-\beta c(t)+p(t-\tau)
$$

We will reassess below the main properties of this discrete delay model before working in Section 3 with the general definition of the measure in order to cover the wider range possible of distribution structures.

\subsection{The impact of a single discrete delay on the optimal pollution path}

The properties of the benchmark problem with initial condition $c(0)=c_{0}$ are well known: the optimal pollution profile has the saddle point property, it is a monotonous trajectory which converges to a unique steady state.

When a discrete delay $\tau$ is taken into account as in (2), the optimal control problem becomes

$$
\begin{aligned}
& \max _{p(.)} \int_{0}^{\infty}[f(\theta p(t))-D(c(t))] e^{-\rho t} d t \\
\text { s.t. } \dot{c}(t)= & -\beta c(t)+p(t-\tau) \\
c(\sigma)= & c_{0}(\sigma) \in \mathcal{C}([-\tau, 0]) \text { given for } \sigma \in[-\tau, 0] \\
p(\sigma)= & p_{0}(\sigma) \in \mathcal{C}^{B}([-\tau, 0)) \text { given for } \sigma \in[-\tau, 0)
\end{aligned}
$$


where $\mathcal{C}([-\tau, 0])$ denotes the set of continuous functions on $[-\tau, 0]$ and $\mathcal{C}^{B}([-\tau, 0))$ denotes the set of continuous functions $x($.$) on [-\tau, 0)$ such that $x\left(0^{-}\right)$is bounded. These additional definitions are standard (d'Albis et al., 2012) as far as the discrete delay case is concerned.

This problem has been studied by Winkler (2008) and Bourgeois and Jayet (2011). As we recall in A with a full resolution, on the optimal path, $c(t)$ depends only on $p(t-\tau)$, for every $t \geq 0$. From the modified system (15) in A.1 we can build a planar phase diagram $(c(t), q(t))$, where $q(t)=p(t-\tau)$, and show that the optimal pollution trajectory has the saddle path property: it converges monotonically to a steady state (see Lemma 7 in A.4).

These results depend highly on the presence of a unique discrete delay and on the separability of the objective function. Indeed, thanks to the separability we can isolate the damage occurring between time 0 and time $\tau$ since this damage is caused by emissions taking place before time 0 and over which the social planner has no control. As $p(\sigma)$ is known for $\sigma \in[-\tau, 0)$, integration of the state equation for $t \in[0, \tau]$ enables us to compute $c(t)$, for $t \in[0, \tau]$. The objective function can thus be rewritten

$$
-\int_{0}^{\tau} D(c(t)) e^{-\rho t} d t+\max _{p(.)} \int_{\tau}^{\infty}\left[f(\theta p(t-\tau)) e^{-\rho(t-\tau)} d t-D(c(t)) e^{-\rho t}\right] d t
$$

The first term corresponds to the damages predetermined before the pollution variable can be controlled. As such, it can be ignored in the maximization program and the problem can be rewritten

$$
\begin{aligned}
& \max _{q(.)} \int_{\tau}^{\infty}\left[f(\theta q(t)) e^{\rho \tau}-D(c(t))\right] e^{-\rho t} d t \\
& \text { s.t. } \dot{c}(t)=-\beta c(t)+q(t) \\
& \text { with initial condition } c(\tau) \text { known }
\end{aligned}
$$

This problem is thus rather similar to the benchmark model except for the weight $e^{\rho \tau}$ on the benefit function. Delays have only quantitative but not qualitative effects on the optimal trajectory. An intuitive result, shown by Winkler (2008) and Bourgeois and Jayet (2011), and for which we present a full proof in A.2 (Lemma 5), is that an increase of the delay increases the optimal steady state pollution stock. The optimality conditions, derived and discussed in A.1 (equation 13 ), allow us to show that a higher delay leads to a higher emission level at the steady state. The economic interpretation of this impact of the delay on the control and the state variables is quite straightforward: because of the delays, the damages caused by the polluting emissions will take place later in time. In a discounted framework this means that these damages will weight less in the social planner's program, who will allow for a higher optimal pollution level at the steady state. It can thus be argued that the delays accelerate the discounting effect on damages while the benefit remain unaffected. This effect will also arise in the case of distributed delays. We complete this result by showing that the dynamics on the optimal path are monotonous (Lemma 6 . 
A.3 and that the saddle property is preserved (Lemma 7. A.4), which is not necessarily the case for distributed delays as we will see in the next section. Figure 1 illustrates this shift of the steady state under the addition of delays that merely "translates" the dynamic system in time.

Combining the positive relation mentioned above between emission level and the delay (Lemma 5 in A.2) and the first order condition (13), it can be easily shown that a higher delay increases the shadow price of pollution $x(t)$ along the optimal path and at the steady state. The increase in the (negative) shadow price caused by the delay implies a reduction in the tax that would implement the optimal pollution path. An optimal environmental policy accounting for delays must be less stringent. Turned around the other way, this indicates that if an environmental policy that ignores delays is implemented it will result in inefficient overregulation. Our result is thus in line with the findings of Kim et al. (1993) and others on the topic.

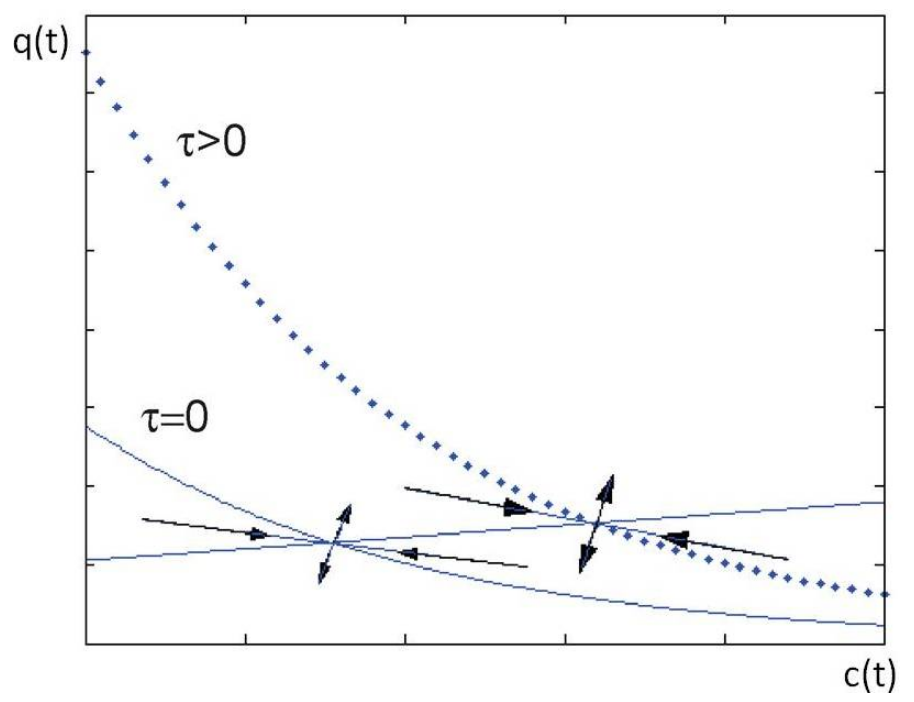

Figure 1: Phase Diagram for the benchmark model $(\tau=0)$ and for a simple delay $(\tau>0)$ in the $(c(t), q(t))$ plane

Overall, the core properties of the benchmark optimal control model are preserved in presence of delays. In terms of environmental policy, this means that the optimal pollution path will be qualitatively similar to the benchmark case but it will result in a higher accumulated pollution stock at the steady state. 


\section{Optimal pollution control in the presence of distributed delays}

Let us now consider the more realistic case of distributed delays. We shall include in our definition of "distributed delays" the case of a sum of discrete delays that can also be captured by our accumulation dynamics (1) and that is presented below. As noted in the Introduction, this configuration can encompass a large range of complex pollution problems characterized by significant site specific heterogeneity in the pollutants'velocity (see Gaines and Gaines, 1994 for the case of nitrates). In order to keep our results as general as possible, we will work with the general definition of the measure presented in Section 2.1. Before solving the general problem, let us give two examples of possible distribution specifications.

\subsection{Examples of specific distribution structures}

To start with, this measure can be specified to reflect a simple case where the initial polluting emission $p(t)$ will reach the stock in two loads, one at time $t+\tau_{1}$ and the other at time $t+\tau_{2}$, with $0 \leq \tau_{1}<\tau_{2}$. Denoting the weight of each load respectively $\varepsilon$ and $(1-\varepsilon)$, with $\varepsilon \in(0,1)$, this mechanism corresponds to a sum of weighted Dirac measures (see Figure 2). In that case the measure function $\mu$ is written

$$
\mu(v)=\varepsilon \delta_{\tau_{1}}(v)+(1-\varepsilon) \delta_{\tau_{2}}(v)
$$

and the accumulation equation (1) becomes a delay differential equation with two discrete delays. More precisely, it can be rewritten

$$
\dot{c}(t)=-\beta c(t)+\left[\varepsilon p\left(t-\tau_{1}\right)+(1-\varepsilon) p\left(t-\tau_{2}\right)\right]
$$

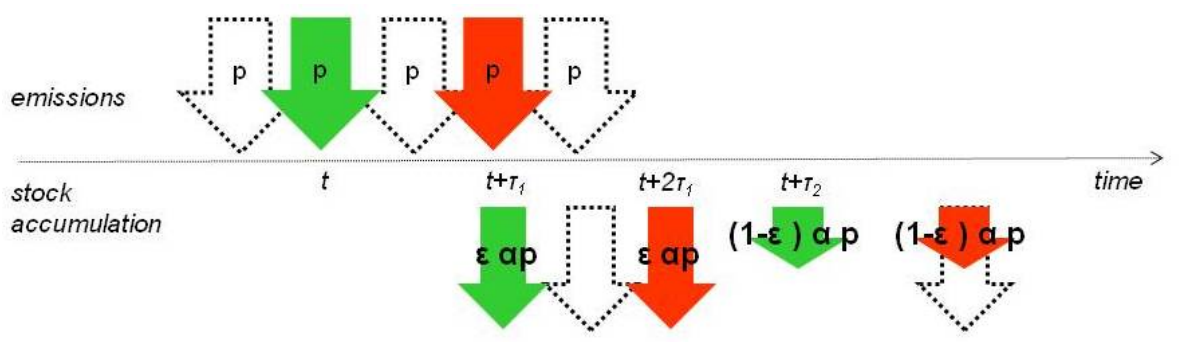

Figure 2: Sum of discrete delays

The general measure function can also be specified as a truncated probability exponential distribution over a time interval $\left[\tau_{1}, \tau_{2}\right]$ such that 


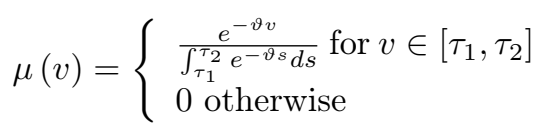

This distribution, roughly illustrated in a discretized manner in Figure 3. can cover a wider range of delayed accumulation mechanisms as the initial load is divided in a continuous distribution over time. The parameter $\vartheta$ sets the repartition of this load in time: the higher $\vartheta$ the earlier the emissions reach the stock within the time interval $\left[\tau_{1}, \tau_{2}\right]$. The accumulation equation (1) now becomes

$$
\dot{c}(t)=-\beta c(t)+\frac{\int_{t-\tau_{2}}^{t-\tau_{1}} e^{-\vartheta(t-s)} p(s) d s}{\int_{\tau_{1}}^{\tau_{2}} e^{-\vartheta s} d s}
$$

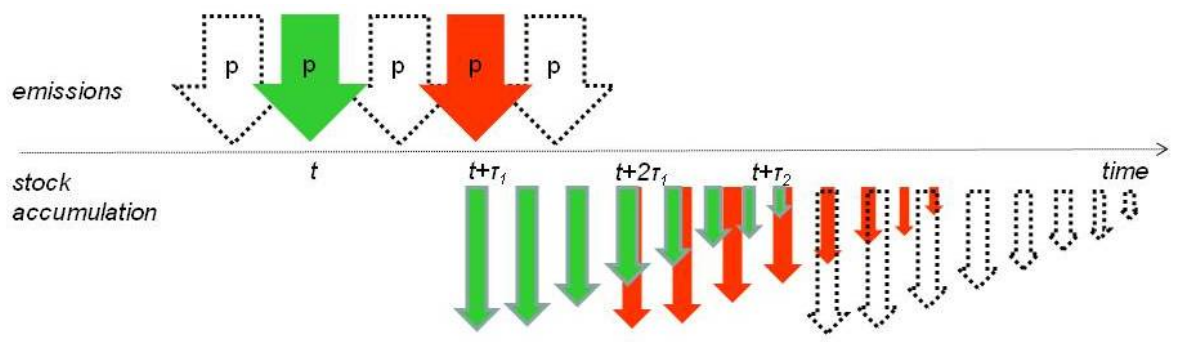

Figure 3: Distributed delays with exponential kernel (discretized representation)

\subsection{Pollution control with distributed delays}

Let us now tackle the model with the general measure function

$$
\begin{aligned}
& \max _{p(.)} \int_{0}^{\infty}[f(\theta p(t))-D(c(t))] e^{-\rho t} d t \\
s c \dot{c}(t)= & -\beta c(t)+\frac{\int_{t-\tau_{2}}^{t-\tau_{1}} p(u) \mu(t-u) d u}{\int_{\tau_{1}}^{\tau_{2}} \mu(u) d u} \\
c(\sigma)= & c_{0}(\sigma) \in \mathcal{C}\left(\left[-\tau_{2}, 0\right]\right) \text { given for } \sigma \in\left[-\tau_{2}, 0\right] \\
p(\sigma)= & p_{0}(\sigma) \in \mathcal{C}^{B}\left(\left[-\tau_{2}, 0\right)\right) \text { given for } \sigma \in\left[-\tau_{2}, 0\right)
\end{aligned}
$$

For the sake of clarity, let us denote $\alpha_{12}=\frac{1}{\int_{\tau_{1}}^{\tau_{2}} \mu(s) d s}$ the constant factor weighting the emissions $\int_{t-\tau_{2}}^{t-\tau_{1}} \mu(t-s) p(s) d s$ that took place over the $\left[t-\tau_{2}, t-\right.$ $\left.\tau_{1}\right]$ interval. Contrary to the single discrete delay model, no change of variables can turn the system into a standard problem without delays. We thus need to detail carefully the Lagrangian to rewrite the system in a tractable form. 
The Lagrangian, with $\xi(t)$ the adjoint variable, is written as

$$
L=\int_{0}^{\infty}[f(\theta p(t))-D(c(t))] e^{-\rho t}+\xi(t)\left(-\dot{c}(t)+\beta c(t)+\alpha_{12} \int_{t-\tau_{2}}^{t-\tau_{1}} p(u) \mu(t-u) d u\right) d t
$$

Doing part integration and applying Fubini's theorem (see B.1) on $L$ yields

$$
\begin{aligned}
& L=\int_{0}^{\infty}[f(\theta p(t))-D(c(t))] e^{-\rho t}+\dot{\xi} c(t)-\xi \beta c(t) \\
& -\alpha_{12} p(t) \int_{t+\tau_{2}}^{t+\tau_{1}} \xi(u) \mu(u-t) d u d t+\xi(0) c(0)-\lim _{t \rightarrow \infty}(\xi(t) c(t)) \\
& +\alpha_{12} \int_{-\tau_{2}}^{0} p(t) \int_{0}^{t+\tau_{2}} \xi(u) \mu(u-t) d u d t-\alpha_{12} \int_{-\tau_{1}}^{0} p(t) \int_{0}^{t+\tau_{1}} \xi(u) \mu(u-t) d u d t
\end{aligned}
$$

We thus get the first order conditions

$$
\left\{\begin{array}{l}
\theta f^{\prime}(\theta p(t)) e^{-\rho t}=-\alpha_{12} \int_{t+\tau_{1}}^{t+\tau_{2}} \xi(u) \mu(u-t) d u \\
\dot{\xi}(t)=D^{\prime}(c(t)) e^{-\rho t}+\xi(t) \beta
\end{array}\right.
$$

and the transversality condition

$$
\lim _{t \rightarrow \infty} \xi(t) c(t)=0
$$

Substituting in the previous system $x(t)=\xi(t) e^{\rho t}$, where $x(t)$ is the current value shadow price of pollution yields

$$
\left\{\begin{array}{l}
\theta f^{\prime}(\theta p(t))=-\alpha_{12} \int_{t+\tau_{1}}^{t+\tau_{2}} x(u) e^{-\rho(u-t)} \mu(u-t) d u \\
\dot{x}(t)=D^{\prime}(c(t))+x(t)(\beta+\rho)
\end{array}\right.
$$

and the transversality condition

$$
\lim _{t \rightarrow \infty} x(t) c(t) e^{-\rho t}=0
$$

The first order condition (3) expresses in a framework with distributed delays the traditional trade-off between the marginal benefit triggered by an additional unit of pollution and the marginal damage caused by this pollution, valued by its shadow price. In the benchmark pollution control model without delays, this trade-off takes place between simultaneous emissions, since the emissions at time $t$ reach the stock at the same instantaneous time $t$. However in the presence of delays, this trade-off compares the marginal benefit obtained from emissions at time $t$ with the damages they cause in the time interval $\left[t+\tau_{1}, t+\tau_{2}\right]$. In order to value the damages caused by the portion $\mu(u-t)$ at each time $u$ during this period we must use the the current value shadow price of pollution at that time, $x(u)$, and discounted accordingly over the period $[t, u]$. Hence the right hand term of (3). 


\subsection{Steady state and sensitivity analysis}

From (3) we get the following expression of $p(t)$

$$
p(t)=\frac{1}{\theta} f^{\prime-1}\left(-\frac{\alpha_{12}}{\theta} \int_{t+\tau_{1}}^{t+\tau_{2}} x(u) e^{-\rho(u-t)} \mu(u-t) d u\right)
$$

which we can substitute in the dynamics to get the following system with both leads and lags

$$
\left\{\begin{array}{l}
\dot{c}(t)=-\beta c(t)+\frac{\alpha_{12}}{\theta} \int_{t-\tau_{2}}^{t-\tau_{1}} f^{\prime-1}\left(-\frac{\alpha_{12}}{\theta} \int_{u+\tau_{1}}^{u+\tau_{2}} x(s) e^{-\rho(s-u)} \mu(s-u) d s\right) \mu(t-u) d u \\
\dot{x}(t)=D^{\prime}(c(t))+x(t)(\beta+\rho)
\end{array}\right.
$$

Lemma 1 There exists a unique steady state $\left(c^{*}, x^{*}\right)$ and

$$
\begin{aligned}
& \frac{d c^{*}}{d \tau_{1}}>0 \\
& \frac{d c^{*}}{d \tau_{2}}>0
\end{aligned}
$$

Proof. According to (5), the steady state satisfies

$$
\left\{\begin{array}{l}
\beta c^{*}=\frac{\alpha_{12}}{\theta} \int_{\tau_{1}}^{\tau_{2}} f^{\prime-1}\left(-\frac{\alpha_{12}}{\theta} \int_{\tau_{1}}^{\tau_{2}} x^{*} e^{-\rho s} \mu(s) d s\right) \mu(u) d u \\
x^{*}=-\frac{D^{\prime}\left(c^{*}\right)}{\beta+\rho}
\end{array}\right.
$$

Given the properties of $f$ and $D$, there exists a unique $c^{*}$ solving system (6), that is to say satisfying

$$
H\left(c ; \tau_{1}, \tau_{2}\right)=0
$$

with

$$
H\left(c ; \tau_{1}, \tau_{2}\right)=\frac{\theta \int_{\tau_{1}}^{\tau_{2}} \mu(v) d u}{\alpha_{12} \int_{\tau_{1}}^{\tau_{2}} e^{-\rho s} \mu(s) d s} f^{\prime}\left(\frac{\theta \beta c}{\alpha_{12}}\right)-\frac{D^{\prime}(c)}{\beta+\rho}
$$

We can thus conclude that the steady state $\left(x^{*}, c^{*}\right)$ exists and is unique.

In addition, applying the implicit function theorem to equation (7) we obtain the following comparative statics results:

$$
\begin{aligned}
& \frac{d c^{*}}{d \tau_{1}}=\frac{-\frac{\theta}{\alpha_{12}} \frac{\mu\left(\tau_{1}\right) \int_{\tau_{1}}^{\tau_{2}} \mu(u) d u\left[e^{-\rho \tau_{1}}-e^{-\rho s}\right] d s}{\left(\int_{\tau_{1}}^{\tau_{2}} e^{-\rho s} \mu(s) d s\right)^{2}} f^{\prime}\left(\frac{\theta \beta c}{\alpha_{12}}\right)}{\frac{\theta \int_{\tau_{1}}^{\tau_{2}} \mu(u) d u}{\alpha_{12} \int_{\tau_{1}}^{\tau_{2}} e^{-\rho s} \mu(s) d s} \frac{\theta \beta}{\alpha_{12}} f^{(2)}\left(\frac{\theta \beta c}{\alpha_{12}}\right)-\frac{D^{(2)}(c)}{\beta+\rho}}>0 \\
& \frac{d c^{*}}{d \tau_{2}}=\frac{-\frac{\theta}{\alpha_{12}} \frac{\mu\left(\tau_{2}\right) \int_{\tau_{1}}^{\tau_{2}}\left(e^{-\rho s}-e^{-\rho \tau_{2}}\right) \mu(s) d s}{\left(\int_{\tau_{1}}^{\tau_{2}} e^{-\rho s} \mu(s) d s\right)^{2}} f^{\prime}\left(\frac{\theta \beta c}{\alpha_{12}}\right)}{\frac{\theta \int_{\tau_{1}}^{\tau_{2}} \mu(u) d u}{\alpha_{12} \int_{\tau_{1}}^{\tau_{2}} e^{-\rho s} \mu(s) d s} \frac{\theta \beta}{\alpha_{12}} f^{(2)}\left(\frac{\theta \beta c}{\alpha_{12}}\right)-\frac{D^{(2)}(c)}{\beta+\rho}}>0
\end{aligned}
$$


Lemma 1 shows that the existence of a unique steady state is preserved and the comparative statics properties of distributed delay on the optimal accumulation are similar to those of the discrete delay case. Whether there is an increase in the lower bound $\tau_{1}$ or the upper bound $\tau_{2}$ of the delay time interval, it will decrease the present value of future damages and favor a higher accumulation of pollution $c^{*}$ according to the accelerated discounting mechanism highlighted in the previous section.

\subsection{Analytical characterization of the dynamics}

\subsubsection{Saddle point configuration and stability}

We are now going to study the local dynamics in the neighborhood of the steady state and determine the conditions under which the latter is a saddle point. For the rest of our analysis we define $B$ such that

$$
B=\left(\frac{\alpha_{12}}{\theta}\right)^{2} \frac{D_{*}^{(2)}}{f_{*}^{(2)}}
$$

where $f_{*}^{(2)}=f_{*}^{(2)}\left(\theta p^{*}\right)$ and $D_{*}^{(2)}=D_{*}^{(2)}\left(c^{*}\right)$.

Our economic interpretation of the dynamic properties of the system will revolve around the (absolute) value of the (negative) parameter $B$.

Linearizing (5) around the steady state yields the following system:

$$
\left\{\begin{array}{l}
\dot{c}(t)=-\beta c-\frac{\left(\alpha_{12}\right)^{2}}{\theta^{2} f_{*}^{(2)}} \int_{t-\tau_{2}}^{t-\tau_{1}} \int_{u+\tau_{1}}^{u+\tau_{2}} x(s) e^{-\rho(s-u)} \mu(s-u) d s \mu(t-u) d u \\
\dot{x}(t)=D_{*}^{(2)} c(t)+x(t)(\beta+\rho)
\end{array}\right.
$$

We compute the characteristic equation $\Delta(\lambda)=0$ of system $(8)$ where $\Delta(\lambda)$ is defined as

$$
\Delta(\lambda)=\operatorname{det}\left(\begin{array}{cc}
\lambda+\beta & \frac{\left(\alpha_{12}\right)^{2}}{\theta^{2} f_{*}^{\prime \prime}} \int_{\tau_{1}}^{\tau_{2}} \mu(s) \int_{\tau_{1}}^{\tau_{2}} e^{\lambda(u-s)} e^{-\rho u} \mu(u) d u d s \\
-D_{*}^{(2)} & \lambda-(\beta+\rho)
\end{array}\right)
$$

Hence

$$
\Delta(\lambda)=(\lambda+\beta)(\lambda-(\beta+\rho))+B \int_{\tau_{1}}^{\tau_{2}} \mu(s) e^{-\lambda s} \int_{\tau_{1}}^{\tau_{2}} e^{-(\rho-\lambda) u} \mu(u) d u d s
$$

The stability properties of the system will depend on the localization of the complex roots of the characteristic equation $\Delta(\lambda)=0$. Characteristic equations of MFDEs are known to have an infinite number of complex isolated roots with positive and negative real part. Moreover, in our case it can be easily proved by replacing $\lambda$ by $(\rho-\lambda)$ in the characteristic equation that these roots are symmetric along the axis $\zeta=\frac{\rho}{2}$. 
We first look at the conditions to have a saddle configuration. In that case there must not be any pure imaginary roots. If pure imaginary roots did exist, by solving equation $\Delta(i q)=0$ and splitting the real and imaginary parts, we would get

$$
\left\{\begin{array}{l}
-q^{2}-(\beta+\rho) \beta+B \int_{\tau_{1}}^{\tau_{2}} \int_{\tau_{1}}^{\tau_{2}} \mu(s) e^{-\rho u} \mu(u) \cos (q(s-u)) d u d s=0(10) \\
-q \rho+B \int_{\tau_{1}}^{\tau_{2}} \int_{\tau_{1}}^{\tau_{2}} \mu(s) e^{-\rho u} \mu(u) \sin (q(u-s)) d u d s=0
\end{array}\right.
$$

Let us state the following conditions:

Condition $1 B>B^{S 1}$ where $B^{S 1}$ is defined as

$$
B^{S 1}=\frac{\rho}{\int_{\tau_{1}}^{\tau_{2}} \int_{\tau_{1}}^{\tau_{2}} \mu(s) e^{-\rho u} \mu(u)(u-s) d u d s-\int_{\tau_{1}}^{\tau_{2}} \int_{s}^{\tau_{2}} \mu(s) e^{-\rho u} \mu(u)[1.218(u-s)] d u d s}
$$

Condition $2 B>B^{S 2}$ where $B^{S 2}$ is defined as

$$
B^{S 2}=\frac{-\left(\left(\left((\beta+\rho) \beta+\frac{\rho^{2}}{2}\right)^{2}+(\beta+\rho) \beta\right)^{2}+\left(\left((\beta+\rho) \beta+\frac{\rho^{2}}{2}\right) \rho\right)^{2}\right)^{\frac{1}{2}}}{\left(\int_{\tau_{1}}^{\tau_{2}} \mu^{2}(s) d s \int_{\tau_{1}}^{\tau_{2}} e^{-2 \rho u} \mu^{2}(u)\right)^{\frac{1}{2}}}
$$

Lemma 2 If $B>B^{S}=\min \left[B^{S 1}, B^{S 2}\right]$, the steady state is a saddle point.

Proof. The proof, given in B.2, relies on the fact that if Condition 1 or Condition 2 holds, $\Delta(\lambda)=0$ has no pure imaginary roots. Since the equation has an infinity of roots with negative and positive real parts, we have a saddle point configuration.

Lemma 2 establishes the existence of saddle configuration. In order to characterize more precisely the saddle property, we can reformulate the problem by noticing that the MFDE in system (5) depends only on the delay $\tau_{1}-\tau_{2}$ and on the advance $\tau_{2}-\tau_{1}$. This property relies on the fact that the problem, as in the single discrete delay setting (see Section 2.2), can be decomposed into two phases. Indeed, knowing $p(\sigma)$ for $\sigma \in\left[-\tau_{2}, 0\right)$ enables us to compute $c(t)$, for $t \in\left[0, \tau_{1}\right]$ and to isolate the corresponding damages over which no control can be exerted. Using the change of variable $p\left(t-\tau_{1}\right)=q(t)$, the objective 
function can then be rewritten,

$$
\begin{aligned}
& \int_{0}^{\tau_{1}}-D(c(t)) e^{-\rho t} d t+\max _{q(.)} \int_{\tau_{1}}^{\infty}\left[f(\theta q(t)) e^{\rho \tau_{1}}-D(c(t))\right] e^{-\rho t} d t \\
& \text { s.t. } \dot{c}(t)=-\beta c(t)+\alpha_{12} \int_{t+\tau_{1}-\tau_{2}}^{t} q(u+t) \mu\left(u-\tau_{1}\right) d u \\
& c(\sigma)=c_{0}(\sigma) \in \mathcal{C}\left(\left[-\tau_{2}+2 \tau_{1}, \tau_{1}\right]\right) \text { given for } \sigma \in\left[-\tau_{2}+2 \tau_{1}, \tau_{1}\right] \\
& q(\sigma)=q_{0}(\sigma) \in \mathcal{C}^{B}\left(\left[-\tau_{2}+2 \tau_{1}, \tau_{1}\right)\right) \text { given for } \sigma \in\left[-\tau_{2}+2 \tau_{1}, \tau_{1}\right)
\end{aligned}
$$

We can thus focus on the second term of the program (12). Although the problem depends highly on initial conditions being given on $\left[-\tau_{2}, 0\right]$ for the state variable and $\left[-\tau_{2}, 0\right)$ for the control variable, the long run dynamic can be reformulated in term of initial conditions on an interval of length $\left(\tau_{2}-\tau_{1}\right)$. Taking this into consideration, the MFDE (5) that arises from the first order conditions of the reformulated problem corresponds to an operator mathematically operating on state $C\left(\left[-\tau_{2}+2 \tau_{1}, \tau_{1}\right]\right)$.

According to our previous conditions which rule out the existence of imaginary roots, there exists two sets $S$ and $U$ such that

$$
S \oplus U=C\left(\left[-\tau_{2}+2 \tau_{1}, \tau_{1}\right]\right)
$$

where $S \subset C\left(\left[-\tau_{2}+2 \tau_{1}, \tau_{1}\right]\right)$ is the set of initial functions leading to convergent solutions as time tends to infinity, and $U \subset C\left(\left[-\tau_{2}+2 \tau_{1}, \tau_{1}\right]\right)$ is the set of initial functions leading to convergent solutions as time tends to minus infinity. This property means that the dynamics can be projected on a stable manifold which is of infinite dimension. The dynamics on the stable manifold can either be monotonous or may display damped oscillations: the following Lemma distinguishes these cases.

Lemma 3 Assuming Condition 1 or Condition 2 hold, there exists a unique scalar $B^{D}$ such that, if $B<B^{D}$, the optimal path displays damped oscillations in the neighborhood of the steady state. Otherwise, the optimal path may be monotonous.

Proof. The proof, detailed in B.3, relies on the demonstration that there exists a unique scalar $B^{D}$ such that $\Delta(\lambda)=0$ has respectively 0 or 4 real roots if $B<B^{D}$ or $B>B^{D}$. The case $B=B^{D}$ is a non-generic case with a root $\lambda_{D}=\lambda\left(B^{D}\right)$ solving $\Delta\left(\lambda_{D}\right)=0$ and $\Delta^{\prime}\left(\lambda_{D}\right)=0$.

Lemma 3 implies that when we study the monotonicity of optimal paths in this complex framework of distributed delays we are faced with two possibilities. If $B<B^{D}$ then the optimal path will display an oscillatory behavior. However if $B>B^{D}$, the optimal path will be characterized by oscillations in the short term but it will eventually converge towards our unique steady state $\left(c^{*}, x^{*}\right)$. In that case, the appropriate shadow price, implemented through a pigovian tax for example, will set the system on the optimal pollution path that will reach the desirable steady state in the long run, despite possible initial oscillations. 


\subsubsection{Pure imaginary roots and Hopf bifurcation}

We have given in Lemma 2 the conditions under which no pure imaginary roots can occur. Let us now study the case where pure imaginary roots exist and the potential consequences in terms of limit cycles.

Let us state the following conditions:

Condition $3 B<B^{H 1}$ where $B^{H 1}$ is defined as

$$
B^{H 1}=\rho\left(\int_{\tau_{1}}^{\tau_{2}} \int_{\tau_{1}}^{\tau_{2}}(u-s) e^{-\rho u} \mu(u) d u d s\right)^{-1}
$$

Condition $4 B<B^{H 2}$ where $B^{H 2}$ is defined as

$$
B^{H 2}=\frac{-(\beta+\rho) \beta}{\left(\int_{\tau_{1}}^{\tau_{2}} \mu(s) d s\right)\left(\int_{\tau_{1}}^{\tau_{2}} e^{-\rho u} \mu(u) d u\right)}
$$

Condition $5 B<B^{H 3}$ where $B^{H 3}$ is defined as

$$
B^{H 3}=-2\left(\int_{\tau_{1}}^{\tau_{2}} \int_{\tau_{1}}^{\tau_{2}}(u-s)^{2} e^{-\rho u} \mu(u) d u d s\right)^{-1}
$$

Lemma 4 The optimal path will give rise to Hopf bifurcations if $B<B^{H}$ where $B^{H}=\min \left[B^{H 1}, B^{H 2}, B^{H 3}\right]$.

Proof. We show in B.4 that if Conditions 34 and 5 hold simultaneously, the conditions for the application of the Hopf bifurcation theorem are met.

Lemma (4) provides an interesting addition to the literature on limit cycles in an infinite dimensional control setting as it sheds some light on the key structural parameters that thought to cause these cycles. In terms of environmental policy, our model shows that if the conditions above hold, a cyclical policy around the steady state will be optimal, alternating pollution accumulation and pollution reduction phases through significant variations of the optimal emission level, implemented in turn by the shadow price of pollution.

\subsection{Discussion}

\section{Consistency of the dynamics characterization}

Let us first verify that our results on the dynamic properties of the optimal pollution path are consistent. It can be easily shown by comparing $\widetilde{\varphi}$ and $\varphi(0)$ that $B^{S 1}>B^{H 1}$. Similarly, using the Jensen inequality we can show that $B^{S 2}>B^{H 2}$. We can thus deduce, that for any value of $B^{H 3}$ 


$$
\min \left[B^{S 1}, B^{S 2}\right]>\min \left[B^{H 1}, B^{H 2}, B^{H 3}\right]
$$

and thus

$$
\begin{aligned}
& B>B^{S} \Rightarrow B>B^{H} \\
& B<B^{H} \Rightarrow B<B^{S}
\end{aligned}
$$

Hence if $B$ is such that the necessary conditions for saddle stability hold, then at least one condition for the occurrence of a Hopf bifurcation does not hold and conversely. We can thus represent on Figure 4 the spectrum of the dynamic behavior of the optimal pollution path depending on the absolute value of $B$.

In terms of environmental policy, a cyclical policy around the steady state will be optimal in the limit cycles regime $\left(|B|>B^{H}\right)$, alternating pollution accumulation and pollution reduction phases through significant variations of the optimal emission level, implemented in turn by the shadow price of pollution. Such limit cycles rarely arise in standard optimal pollution control problem, except in the presence of non-convexities (Tahvonen and Salo, 1996) or adjustment costs (Wirl, 1999).

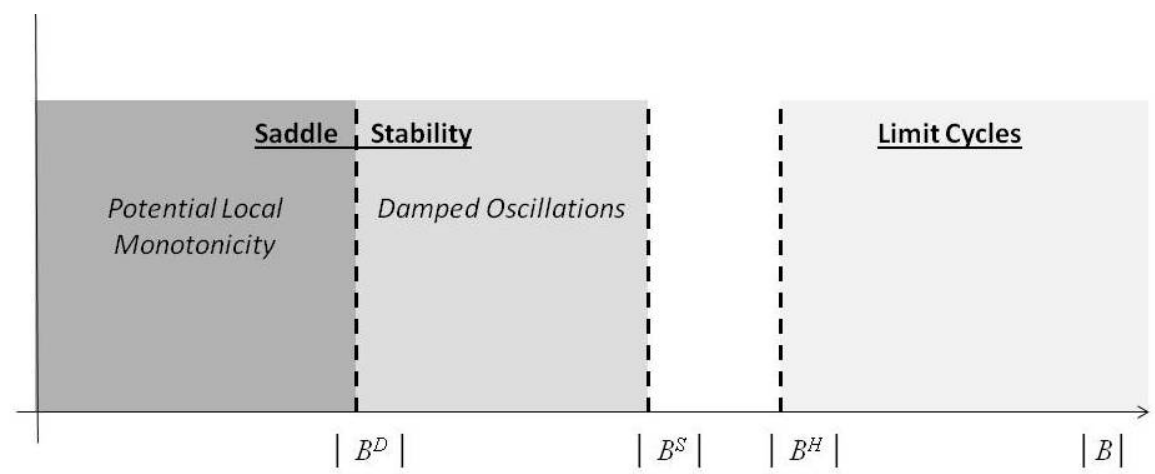

Figure 4: Dynamic Properties of the optimal path depending on $|B|$

\section{Discussion of the determinants of $B$}

In order to discuss the operational power of our model let us discuss the features that determine the regime of the optimal path. The latter depends heavily on the absolute value of $B$. To keep our economic interpretation as clear as possible, we shall start by distinguishing within $B$ two components: the technological ratio $\frac{\alpha_{12}}{\theta}$ and the preference ratio $\frac{D_{*}^{(2)}}{f_{*}^{(2)}}$. 
Technological ratio It is straightforward that a higher $\theta$ leads to a smaller $|B|$. As a result, optimal policies derived from our model will be (locally) monotonous ceteris paribus in a setting where the polluting byproduct of the economic activity is high, and as this technology improves, the likelihood of oscillations, and then of limit cycles, increases.

The second component of the technological ratio is $\alpha_{12}=\frac{1}{\int_{\tau_{1}}^{\tau_{2}} \mu(s) d s}$. For a given measure $\mu$, a contraction (respectively an extension) of the delay interval $\left[\tau_{1}, \tau_{2}\right]$ through either bound will lead to a higher (smaller) $|B|$. The occurrence of limit cycles, and, to a smaller extent, of damped oscillations, will thus be less likely if the time-lag interval is long. It could be argued that this effect is due to the smoothing effect of a broad distribution of delayed emissions in time that eventually levels out the oscillations that could arise in more condensed time interval. This effect is an interesting addition to the determinants of limit cycles discussed in the literature. Indeed, the focus on short term, via a high discount rate, has been highlighted by Dockner and Feichtinger (1991) as a favorable condition for the arising of optimal limit cycles and our result confirms this finding in a different perspective.

Preference ratio In order to relate more easily our results with the literature on limit cycles, we will consider our preference ratio $\frac{D_{*}^{(2)}}{f_{*}^{(2)}}$ as an indicator of the concavity of our (separable) objective function. High absolute values for this ratio, that is to say a highly convex damage function and/or a highly concave benefit function, reflect weakly green social preferences that attribute a significant value to marginal environmental damages but are not so strong as to forbid any pollution at all. These weakly green preferences have been identified by Wirl (1999) in a model without delays as potential determinants of limit cycles in two dimensional control problems. Our analytical characterization thus complete these previous results by identifying the conditions of occurrence of limit cycles in infinite dimension control problems complexified by the introduction of distributed delays that disturb the time frame of the model. If the preferences are less green, then the optimal policy will be stable, although it might involve damped oscillations.

If we separate the sources of the concavity of our separable objective function, we observe that the case corresponding to a monotonous optimal policy is the case of a damage function that is not too convex and a benefit function that is concave enough. This situation fits quite well various cases of pollution when the economic yield of the polluters depends only partially on the amount of pollution emitted and when the damages do not increase too steeply with the stock. For example in the case of nitrate contamination we can consider that the most important part of the damages is done once the stock has reached the threshold that makes drinking water improper for consumption or that triggers eutrophication of water bodies. 


\section{Conclusion}

We have developed an original characterization of a little known class of infinite dimension optimal control models including distributed delays and a separable objective function. Our work highlights the need to account for the significant time delays that can be involved in pollution accumulation problems. Mod-

eling explicitly these time delays allows to reflect the spatial heterogeneity or the disturbances in the accumulation processes that characterize various pollution problems. These delays might explain why some recent environmental policies targeting pollution reduction, in the field of nitrate regulation for example, have failed to show significant results yet. But their presence in the standard pollution control model modifies quantitatively and qualitatively the optimal pollution path properties and gives rise to complex dynamics. From a quantitative standpoint we have shown that the greater the delay, the higher the pollution stock at equilibrium and the less stringent the optimal environmental tax. From a qualitative perspective, the dynamic properties of the model are deeply disturbed because MFDEs are involved.

To face this challenge, we have developed a method that allows us, while preserving generic functional forms and a very general distribution structure, to assess the main properties of the optimal path and the conditions of stability and oscillations. Our analysis has led us in particular to determine the conditions of occurrence of limit cycles, which are rather original for this kind of standard pollution control problem. Our results enrich the literature on limit cycles by extending Wirl's conclusions on the role of weakly green preferences.

Our contribution sets a robust ground that can be extended to a wider range of economic problems beyond the realm of pollution control. We trust it can help to address better the analytical difficulties at stake in many dynamic economic setting where complex delays play a significant role such as capital accumulation or advertising policies. 


\section{Acknowledgements}

The authors are grateful for financing from ADAPT'EAU, a project supported by the ANR, and from the CNRS through the PEPS initiative. The authors would like to thank participants at the SURED 2012 conference and at the AERNA 2012 congress for helpful comments. This work originated from stimulating discussions during the M3D network 2011 Spring School.

\section{References}

Bordenave, P., Bouraoui, F., Gascuel-Odoux, C., Molénat, J., Mérot, P., 1999, Décalages temporels entre des modifications des pratiques agricoles et la diminution des nitrates dans les eaux superficielles. In: Merceron, M. (Ed), Pollutions diffuses : du bassin au littoral (IFREMER) vol. 24, pp. 311-333.

Bourgeois, C., Jayet, P., 2011. Revisited water-oriented relationships between a set of farmers and an aquifer: accounting for lag effect. Working Paper. http:// WWW.grignon.inra.fr/economie-publique/docs_travail/docs_2010/2010_ 06.pdf

Boucekkine, R., Fabbri, G. Gozzi, F., 2010. Maintenance and investment: complements or substitutes? A reappraisal. Journal of Economic Dynamics and Control 34, 2420-2439.

d'Albis, H., Augeraud-Veron, E., Hupkes, H.J., 2012. Discontinuous initial value problems for functional differential-algebraic equations of mixed type. Working Paper. http://halshs.archives-ouvertes.fr/halshs-00717412

Federico, S., Goldys, B., Gozzi, F., 2010. HJB Equations for the optimal control of differential equations with delays and state constraints: regularity of viscosity solutions. Siam Journal of Control Optimization 48(8), 4910-4937.

Gaines, T.P., Gaines, S.T., 1994. Soil texture effect on nitrate leaching in soil percolates. Communications in Soil Science and Plant Analysis 25(13\&14), 2561-2570.

Grimvall, A., Stälnacke, P., Tonderski, A., 2000. Time scales of nutrient losses from land to sea-a European perspective. Ecological Engineering 14(4), 363-371.

Keeler. E., Spence M., Zeckhauser, R., 1972. The optimal control of pollution. Journal of Economic Theory 4, 19-34.

Kim, C., Hostetler, J., Amacher, G., 1993. The regulation of groundwater quality with delayed responses. Water Resources Research 29(5), 1369-1377.

Thavonen, O., Salo., S., 1996. Nonconvexities in optimal pollution accumulation. Journal of Environmental Economics and Management 31, 160-177.

Winkler, R., 2008. Optimal control of pollutants with delayed stock accumulation. CER-ETH Working Paper 08/91.

Winkler, R., 2011. A Note on the optimal control of stocks accumulating with a delay. Macroeconomic Dynamics 15, 565-578. 
Wirl, F., 1999. Complex dynamic environmental policies. Resource and Energy Economics 21, 19-41.

\section{A Single discrete delay model}

\section{A.1 Preliminary results}

With constant time lags $\tau$ between nitrate emissions and nitrate accumulation the motion equation is now written: $\dot{c}(t)=-\beta c(t)+p(t-\tau)$. We shall solve the new program faced by the social planner in a different manner than Bourgeois and Jayet (2011) that will be useful to solve the general case of distributed delays.

$$
\begin{aligned}
& \max _{p(.)} \int_{0}^{\infty}[f(\theta p(t))-D(c(t))] e^{-\rho t} d t \\
\text { s.t. } \dot{c}(t)= & -\beta c(t)+p(t-\tau) \\
c(\sigma)= & c_{0}(\sigma) \in \mathcal{C}([-\tau, 0]) \text { given for } \sigma \in[-\tau, 0] \\
p(\sigma)= & p_{0}(\sigma) \in \mathcal{C}^{B}([-\tau, 0)) \text { given for } \sigma \in[-\tau, 0)
\end{aligned}
$$

The Hamiltonian is

$$
[f(\theta p(t))-D(c(t))] e^{-\rho t}+\xi(t)[-\beta c(t)+p(t-\tau)]
$$

Given that the Hamiltonian is concave, we have the following first order conditions, with $x(t)=\xi(t) e^{\rho t}$ the current value shadow price of pollution along the optimal path.

$$
\begin{gathered}
\theta f^{\prime}(\theta p(t))=-x(t+\tau) e^{-\rho \tau} \\
\dot{x}(t)=D^{\prime}(c(t))+x(t)(\beta+\rho)
\end{gathered}
$$

and we have the transversality condition

$$
\lim _{t \rightarrow \infty} c(t) x(t) e^{-\rho t}=0
$$

Deriving (13) with respect to time $t$ and combining with (14) yields

$$
\left\{\begin{array}{l}
\dot{p}(t)=\frac{\frac{d-\left(x(t+\tau) e^{-\rho \tau}\right)}{d t}}{\theta^{2} f^{(2)}(\theta p(t))} \\
\dot{p}(t)=\frac{-\left(D^{\prime}(c(t+\tau))+x(t+\tau)(\beta+\rho)\right) e^{-\rho \tau}}{\theta^{2} f^{(2)}(\theta p(t))}
\end{array}\right.
$$

With the change of variable $p(t-\tau)=q(t)$ and the substitution of $x(t+\tau)$ by its value $-\frac{\theta}{1} f^{\prime}(\theta p(t)) e^{\rho \tau}$ in 14 , the system depending on the variables $(c(t), p(t))$ 
becomes a new system in terms of $(c(t), q(t))$ where the dynamics of $c(t)$ and $q(t)$ involve only variables taken at time $t$.

$$
\left\{\begin{array}{l}
\dot{q}(t)=\frac{-e^{-\rho \tau} D^{\prime}(c(t))+\theta f^{\prime}(\theta q(t))(\beta+\rho)}{\theta^{2} f^{(2)}(\theta q(t))} \\
\dot{c}(t)=-\beta c(t)+q(t)
\end{array}\right.
$$

which allows us to draw the phase diagram presented in Figure 1 and to prove the following Lemmas.

\section{A.2 Proof of Lemma 5}

Lemma 5 There is a unique steady state $\left(c^{*}, q^{*}\right)$ and the higher the delay, the higher the stock of pollution (and consequently the higher the emission level) at the steady state

From (15) we know that the steady state solves

$$
\left\{\begin{array}{l}
\beta c=q \\
e^{-\rho \tau} D^{\prime}\left(\frac{q}{\beta}\right)=\theta f^{\prime}(\theta q)(\beta+\rho)
\end{array}\right.
$$

Given the properties of $f$ and $D$, we can establish that $e^{-\rho \tau} D^{\prime}\left(\frac{q}{\beta}\right)$ is an increasing function of $q$ and that $\theta f^{\prime}(\theta q)(\beta+\rho)$ is a decreasing function of $q$. Therefore there exists a unique $q^{*}$ that solves (16), and a unique steady state $\left(c^{*}, q^{*}\right)$.

Let us consider $F(q, \tau)=e^{-\rho \tau} D^{\prime}\left(\frac{q}{\beta}\right)-\theta f^{\prime}(\theta q)(\beta+\rho)$. As $F_{q}^{\prime} \neq 0$, we can apply the implicit function theorem and get

$$
\begin{aligned}
\frac{d q_{\tau}^{*}}{d \tau} & =\frac{\rho e^{-\rho \tau} D^{\prime}\left(\frac{q^{*}}{\beta}\right)}{\frac{1}{\beta} e^{-\rho \tau} D^{(2)}\left(\frac{q}{\beta}\right)-\theta^{2} f^{(2)}(\theta q)(\beta+\rho)}>0 \\
\frac{d c_{\tau}^{*}}{d \tau} & =\frac{\rho \frac{1}{\beta} e^{-\rho \tau} D^{\prime}\left(\frac{q}{\beta}\right)}{\frac{1}{\beta} e^{-\rho \tau} D^{(2)}\left(\frac{q}{\beta}\right)-\theta^{2} f^{(2)}(\theta q)(\beta+\rho)}>0
\end{aligned}
$$

\section{A.3 Proof of Lemma 6}

Lemma 6 Dynamics on the optimal path are monotonous

Global analysis:

The isocline $\dot{p}=0$ is the graph of a function $\psi_{p}(p)=c$ with

$$
\psi_{p}^{\prime}(p)=\frac{\theta^{2} f^{(2)}(\theta p)(\beta+\rho)}{e^{-\tau \rho} D^{(2)}(c)}<0
$$

and the isocline $\dot{c}=0$ is the graph of a function $\psi_{c}(p)=c$, with $\psi_{c}=\frac{1}{\beta} p$. 


\section{A.4 Proof of Lemma 7}

Lemma 7 The steady state is a saddle point. The eigenvalues of the Jacobian related to system (16) are $\lambda_{ \pm}=\frac{\rho \pm \sqrt{\rho^{2}+4\left(\beta(\beta+\rho)-\frac{e^{-\rho \tau} D_{*}^{(2)}}{\theta^{2} f_{*}^{(2)}}\right)}}{2}$.

The Jacobian matrix computed in the neighborhood of the steady state can be written

$$
\left\{\begin{array}{l}
\dot{q}(t)=\frac{-e^{-\rho \tau} D^{(2)}\left(c_{\tau}^{*}\right)}{\theta^{2} f^{(2)}(\theta q(t))} c(t)+(\beta+\rho) q(t) \\
\dot{c}(t)=-\beta c(t)+q(t)
\end{array}\right.
$$

The characteristic polynomial is thus

$$
X^{2}-\rho X-\left(\beta(\beta+\rho)-e^{-\rho \tau} \frac{D_{*}^{(2)}}{\theta^{2} f_{*}^{(2)}}\right)
$$

The steady state admits eigenvalues $\lambda_{-}<0<\lambda_{+}$of opposite signs, therefore it is a saddle point. These eigenvalues are defined as:

$$
\lambda_{ \pm}=\frac{\rho \pm \sqrt{\rho^{2}+4\left(\beta(\beta+\rho)-\frac{e^{-\rho \tau} D_{*}^{(2)}}{\theta^{2} f_{*}^{(2)}}\right)}}{2}
$$

\section{B Distributed delays model}

\section{B.1 Application of Fubini's theorem}

$$
\begin{aligned}
& \int_{0}^{\infty} \xi(t) \int_{t-\tau_{2}}^{t-\tau_{1}} p(u) \mu(t-u) d u d t \\
= & \int_{0}^{\infty} \xi(t) \int_{t-\tau_{2}}^{0} p(u) \mu(t-u) d u d t+\int_{0}^{\infty} \xi(t) \int_{0}^{t-\tau_{1}} p(u) \mu(t-u) d u d t \\
= & \int_{0}^{\tau_{2}} \xi(t) \int_{t-\tau_{2}}^{0} p(u) \mu(t-u) d u d t-\int_{\tau_{2}}^{\infty} \xi(t) \int_{0}^{t-\tau_{2}} p(u) \mu(t-u) d u d t \\
& -\int_{0}^{\tau_{1}} \xi(t) \int_{t-\tau_{1}}^{0} p(u) \mu(t-u) d u d t+\int_{\tau_{1}}^{\infty} \xi(t) \int_{0}^{t-\tau_{1}} p(u) \mu(t-u) d u d t \\
= & \int_{-\tau_{2}}^{0} p(u) \int_{0}^{u+\tau_{2}} \xi(t) \mu(t-u) d t d u-\int_{0}^{\infty} p(u) \int_{u+\tau_{2}}^{\infty} \xi(t) \mu(t-u) d t d u \\
& -\int_{-\tau_{1}}^{0} p(u) \int_{0}^{u+\tau_{1}} \xi(t) \mu(t-u) d t d u+\int_{0}^{\infty} p(u) \int_{u+\tau_{1}}^{\infty} \xi(t) \mu(t-u) d t d u
\end{aligned}
$$




$$
\begin{aligned}
& \int_{0}^{\infty} \xi(t) \int_{t-\tau_{2}}^{t-\tau_{1}} p(u) \mu(t-u) d u d t \\
= & \int_{-\tau_{2}}^{0} p(u) \int_{0}^{u+\tau_{2}} \xi(t) \mu(t-u) d t d u-\int_{-\tau_{1}}^{0} p(u) \int_{0}^{u+\tau_{1}} \xi(t) \mu(t-u) d t d u \\
& +\int_{0}^{\infty} p(u) \int_{u+\tau_{1}}^{u+\tau_{2}} \xi(t) \mu(t-u) d t d u
\end{aligned}
$$

\section{B.2 Proof of Lemma 2}

As the characteristic equation we consider corresponds to an MFDE, there is an infinite number of complex roots with positive real part and negative real part. In order to prove that the steady state is a saddle point we thus need to prove that the characteristic equation admits no pure imaginary roots if either Condition 1 or Condition 2 hold.

\section{Condition 1}

A necessary condition to have a pure imaginary root is that equation $\operatorname{Im} \Delta(i q)=$ 0 is solved, which corresponds to equation (11). This equation can be re-written as:

$$
\frac{\rho}{B}=\varphi(q)
$$

with

$$
\varphi(q)=\frac{\int_{\tau_{1}}^{\tau_{2}} \int_{\tau_{1}}^{\tau_{2}} \mu(s) e^{-\rho u} \mu(u) \sin (q(u-s)) d u d s}{q}
$$

We now would like to prove that $\varphi(q)$ is greater than some (negative) constant $\widetilde{\varphi}$.

$$
\varphi(q)=\frac{\int_{\tau_{1}}^{\tau_{2}} \int_{\tau_{1}}^{s} \mu(s) e^{-\rho u} \mu(u) \sin (q(u-s)) d u d s}{q}+\frac{\int_{\tau_{1}}^{\tau_{2}} \int_{s}^{\tau_{2}} \mu(s) e^{-\rho u} \mu(u) \sin (q(u-s)) d u d s}{q}
$$

Moreover, if $u-s<0$, we have $\frac{\sin (q(u-s))}{q}>(u-s)$. The previous expression can

thus be rewritten as

$$
\begin{aligned}
\varphi(q) & >\int_{\tau_{1}}^{\tau_{2}} \int_{\tau_{1}}^{s} \mu(s) e^{-\rho u} \mu(u)(u-s) d u d s+\frac{\int_{\tau_{1}}^{\tau_{2}} \int_{s}^{\tau_{2}} \mu(s) e^{-\rho u} \mu(u) \sin (q(u-s)) d u d s}{q} \\
& >\int_{\tau_{1}}^{\tau_{2}} \int_{\tau_{1}}^{\tau_{2}} \mu(s) e^{-\rho u} \mu(u)(u-s) d u d s+\int_{\tau_{1}}^{\tau_{2}} \int_{s}^{\tau_{2}} \mu(s) e^{-\rho u} \mu(u)\left[\frac{\sin (q(u-s))}{q}-(u-s)\right] d u d s
\end{aligned}
$$

It is known that $\frac{\sin (q)}{q}>-0.218$. We thus deduce that if $u-s>0$, then $\frac{\sin (q(u-s))}{q}>$

$-0.218(u-s)$. Hence

$$
\begin{aligned}
\varphi(q) & >\int_{\tau_{1}}^{\tau_{2}} \int_{\tau_{1}}^{\tau_{2}} \mu(s) e^{-\rho u} \mu(u)(u-s) d u d s-\int_{\tau_{1}}^{\tau_{2}} \int_{s}^{\tau_{2}} \mu(s) e^{-\rho u} \mu(u)[1.218(u-s)] d u d s \\
& >\varphi(0)-\int_{\tau_{1}}^{\tau_{2}} \int_{s}^{\tau_{2}} \mu(s) e^{-\rho u} \mu(u)[1.218(u-s)] d u d s
\end{aligned}
$$


and since

$$
\begin{aligned}
\varphi(0) & =\int_{\tau_{1}}^{\tau_{2}} \int_{\tau_{1}}^{\tau_{2}} \mu(s) e^{-\rho u} \mu(u)(u-s) d u d s \\
& =\int_{\tau_{1}}^{\tau_{2}} \mu(s) d s \int_{\tau_{1}}^{\tau_{2}} e^{-\rho u} u \mu(u) d u-\int_{\tau_{1}}^{\tau_{2}} \mu(u) u d s \int_{\tau_{1}}^{\tau_{2}} e^{-\rho u} \mu(u) d u \\
& <\int_{\tau_{1}}^{\tau_{2}} \mu(s) d s \int_{\tau_{1}}^{\tau_{2}} e^{-\rho u} u \mu(u) d u-\int_{\tau_{1}}^{\tau_{2}} \mu(u) u d s \int_{\tau_{1}}^{\tau_{2}} \mu(u) d u \\
& <\int_{\tau_{1}}^{\tau_{2}} \mu(s) d s \int_{\tau_{1}}^{\tau_{2}}\left(e^{-\rho u}-1\right) u \mu(u) d u \\
& <0
\end{aligned}
$$

we have

$$
\widetilde{\varphi}=\varphi(0)-\int_{\tau_{1}}^{\tau_{2}} \int_{s}^{\tau_{2}} \mu(s) e^{-\rho u} \mu(u)[1.218(u-s)] d u d s<0 .
$$

Thus if $\frac{\rho}{B}<\widetilde{\varphi}$, equation $\frac{\rho}{B}=\varphi(q)$ has no roots and therefore the characteristic equation has no pure imaginary roots. This condition amounts to $B>B^{S 1}$.

\section{Condition 2}

Using trigonometric forms, system (1011), which supposes the existence of pure imaginary roots, can be written as

$$
\left\{\begin{array}{c}
-q^{2}-(\beta+\rho) \beta+B\left(\begin{array}{c}
\int_{\tau_{1}}^{\tau_{2}} \mu(s) \cos (q s) d s \int_{\tau_{1}}^{\tau_{2}} e^{-\rho u} \cos (q u) \mu(u) \\
+\int_{\tau_{1}}^{\tau_{2}} \mu(s) \sin (q s) d s \int_{\tau_{1}}^{\tau_{2}} e^{-\rho u} \sin (q u) \mu(u) d u
\end{array}\right)=0 \\
-q \rho+B\left(\begin{array}{c}
-\int_{\tau_{1}}^{\tau_{2}} \mu(s) \sin (q s) d s \int_{\tau_{1}}^{\tau_{2}} e^{-\rho u} \cos (q u) \mu(u) d u \\
+\int_{\tau_{1}}^{\tau_{2}} \mu(s) \cos (q s) d s \int_{\tau_{1}}^{\tau_{2}} e^{-\rho u} \sin (q u) \mu(u) d u
\end{array}\right)=0
\end{array}\right.
$$

and

$$
\left\{\begin{array}{c}
q^{2}+(\beta+\rho) \beta=B\left(\begin{array}{c}
\int_{\tau_{1}}^{\tau_{2}} \mu(s) \cos (q s) d s \int_{\tau_{1}}^{\tau_{2}} e^{-\rho u} \cos (q u) \mu(u) d u \\
+\int_{\tau_{1}}^{\tau_{2}} \mu(s) \sin (q s) d s \int_{\tau_{1}}^{\tau_{2}} e^{-\rho u} \sin (q u) \mu(u) d u
\end{array}\right) \\
q \rho=B\left(\begin{array}{c}
-\int_{\tau_{1}}^{\tau_{2}} \mu(s) \sin (q s) d s \int_{\tau_{1}}^{\tau_{2}} e^{-\rho u} \cos (q u) \mu(u) d u \\
+\int_{\tau_{1}}^{\tau_{2}} \mu(s) \cos (q s) d s \int_{\tau_{1}}^{\tau_{2}} e^{-\rho u} \sin (q u) \mu(u) d u
\end{array}\right)
\end{array}\right.
$$


Squaring up both sides of the real and imaginary part, we get

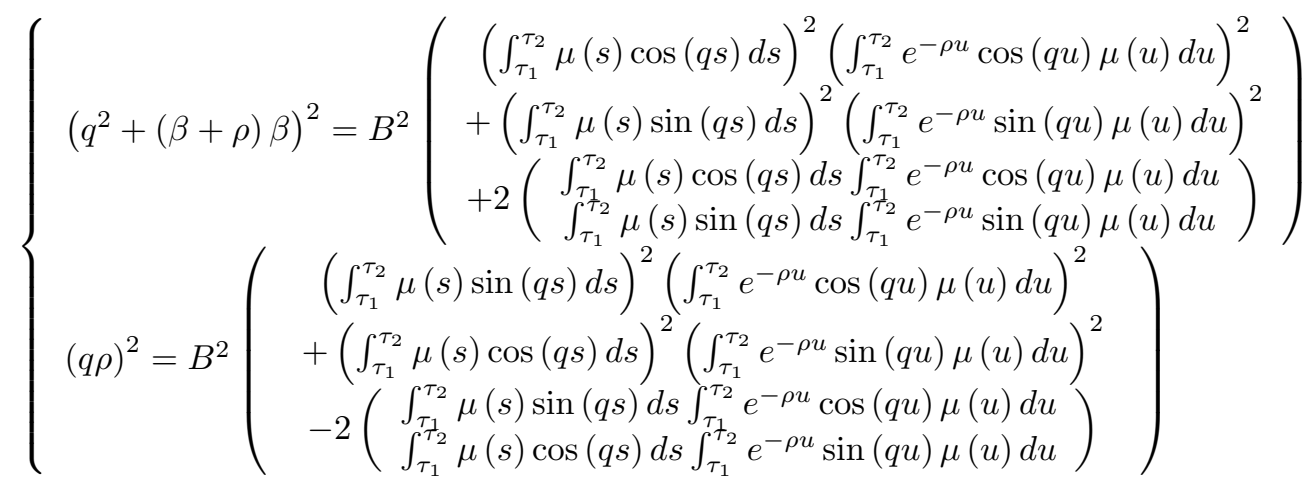

Summing both expressions yields

$$
\left(q^{2}+(\beta+\rho) \beta\right)^{2}+(q \rho)^{2}=B^{2}\left(\begin{array}{c}
{\left[\left(\int_{\tau_{1}}^{\tau_{2}} \mu(s) \cos (q s) d s\right)^{2}+\left(\int_{\tau_{1}}^{\tau_{2}} \mu(s) \sin (q s) d s\right)^{2}\right] *} \\
{\left[\left(\int_{\tau_{1}}^{\tau_{2}} e^{-\rho u} \cos (q u) \mu(u) d u\right)^{2}+\left(\int_{\tau_{1}}^{\tau_{2}} e^{-\rho u} \sin (q u) \mu(u) d u\right)^{2}\right]}
\end{array}\right)
$$

Let us define $P(q)$ such that

$$
P(q)=\left(q^{2}+(\beta+\rho) \beta\right)^{2}+(q \rho)^{2}-B^{2}\left(\begin{array}{c}
{\left[\left(\int_{\tau_{1}}^{\tau_{2}} \mu(s) \cos (q s) d s\right)^{2}+\left(\int_{\tau_{1}}^{\tau_{2}} \mu(s) \sin (q s) d s\right)^{2}\right] *} \\
{\left[\left(\int_{\tau_{1}}^{\tau_{2}} e^{-\rho u} \cos (q u) \mu(u) d u\right)^{2}+\left(\int_{\tau_{1}}^{\tau_{2}} e^{-\rho u} \sin (q u) \mu(u) d u\right)^{2}\right]}
\end{array}\right)
$$

Equation 18 that gives us a necessary condition for having pure imaginary roots can then be rewritten

$$
P(q)=0
$$

We can consider the function $Q$ such that

$$
Q(q)=\left(q^{2}+(\beta+\rho) \beta\right)^{2}+(q \rho)^{2}-B^{2} \int_{\tau_{1}}^{\tau_{2}} \mu^{2}(s) d s \int_{\tau_{1}}^{\tau_{2}} e^{-2 \rho u} \mu^{2}(u)
$$

and since

$$
\left(\begin{array}{l}
{\left[\left(\int_{\tau_{1}}^{\tau_{2}} \mu(s) \cos (q s) d s\right)^{2}+\left(\int_{\tau_{1}}^{\tau_{2}} \mu(s) \sin (q s) d s\right)^{2}\right] *} \\
{\left[\left(\int_{\tau_{1}}^{\tau_{2}} e^{-\rho u} \cos (q u) \mu(u) d u\right)^{2}+\left(\int_{\tau_{1}}^{\tau_{2}} e^{-\rho u} \sin (q u) \mu(u) d u\right)^{2}\right]}
\end{array}\right)<\int_{\tau_{1}}^{\tau_{2}} \mu^{2}(s) d s \int_{\tau_{1}}^{\tau_{2}} e^{-2 \rho u} \mu^{2}(u)
$$

we have

$$
P(q)>Q(q)
$$

$Q$ is a quadratic function in $q^{2}$. Let us denote $Q_{\min }=\min _{q} Q(q)$. If $Q_{\min }>0$, there exist no root $q$ such that $Q(q)=0$, and thus no root such that $P(q)=0$. 
$Q_{\min }$ can be computed as

$$
\begin{aligned}
Q_{\min } & =Q\left((\beta+\rho) \beta+\frac{\rho^{2}}{2}\right) \\
& =\left(\left((\beta+\rho) \beta+\frac{\rho^{2}}{2}\right)^{2}+(\beta+\rho) \beta\right)^{2}+\left(\left((\beta+\rho) \beta+\frac{\rho^{2}}{2}\right) \rho\right)^{2}-B^{2} \int_{\tau_{1}}^{\tau_{2}} \mu^{2}(s) d s \int_{\tau_{1}}^{\tau_{2}} e^{-2 \rho u} \mu^{2}(u)
\end{aligned}
$$

Hence $Q_{\min }>0$ implies

$$
\frac{\left(\left((\beta+\rho) \beta+\frac{\rho^{2}}{2}\right)^{2}+(\beta+\rho) \beta\right)^{2}+\left(\left((\beta+\rho) \beta+\frac{\rho^{2}}{2}\right) \rho\right)^{2}}{\int_{\tau_{1}}^{\tau_{2}} \mu^{2}(s) d s \int_{\tau_{1}}^{\tau_{2}} e^{-2 \rho u} \mu^{2}(u)}>B^{2}
$$

which is tantamount to Condition 2 since $B$ is negative by definition.

\section{B.3 Proof of Lemma 3}

Let us focus on the real roots of $\Delta(\lambda)=0$. As the roots are symmetric according to the line $\operatorname{Re}(\lambda)=\frac{r h o}{2}$ axis, we can study exclusively the roots with a real part smaller than $\frac{r h o}{2}$.

We start by noticing that $\Delta\left(\frac{\rho}{2}\right)<0$, and $\lim _{\lambda \rightarrow \infty} \Delta(\lambda)=-\infty$

Since

$$
\Delta(\lambda)=(\lambda+\beta)(\lambda-(\beta+\rho))+B \int_{\tau_{1}}^{\tau_{2}} \mu(s) e^{-\lambda s} \int_{\tau_{1}}^{\tau_{2}} e^{-(\rho-\lambda) u} \mu(u) d u d s
$$

its derivative according to $\lambda$ is given by

$$
\Delta^{\prime}(\lambda)=2 \lambda-\rho+B \int_{\tau_{1}}^{\tau_{2}} \mu(s) e^{-\frac{\rho}{2} s} \int_{\tau_{1}}^{\tau_{2}}(u-s) e^{-\frac{\rho}{2}(u-s)} e^{-\frac{\rho}{2} u} e^{\lambda(u-s)} \mu(u) d u d s
$$

We notice that

$$
\int_{\tau_{1}}^{\tau_{2}} \mu(s) e^{-\frac{\rho}{2} s} \int_{\tau_{1}}^{\tau_{2}}(u-s) e^{-\frac{\rho}{2} u} \mu(u) d u d s=0
$$

Hence

$$
\Delta^{\prime}(\lambda)=2\left(\lambda-\frac{\rho}{2}\right)+B \int_{\tau_{1}}^{\tau_{2}} \mu(s) e^{-\frac{\rho}{2} s} \int_{\tau_{1}}^{\tau_{2}}(u-s) e^{-\frac{\rho}{2} u}\left[e^{\left(\lambda-\frac{\rho}{2}\right)(u-s)}-1\right] \mu(u) d u d s
$$

Therefore roots of $\Delta^{\prime}(\lambda)=0$ are $\frac{\rho}{2}$ and roots of $\varphi(\lambda)=0$, where $\varphi$ is given by:

$$
\varphi(\lambda)=2+B \int_{\tau_{1}}^{\tau_{2}} \mu(s) e^{-\frac{\rho}{2} s} \int_{\tau_{1}}^{\tau_{2}}(u-s) e^{-\frac{\rho}{2} u}\left[\int_{0}^{(u-s)} e^{\left(\lambda-\frac{\rho}{2}\right) z} d z\right] \mu(u) d u d s
$$


Moreover:

$$
\varphi^{(2)}(\lambda)=B \int_{\tau_{1}}^{\tau_{2}} \mu(s) e^{-\frac{\rho}{2} s} \int_{\tau_{1}}^{\tau_{2}}(u-s) e^{-\frac{\rho}{2} u}\left[\int_{0}^{(u-s)} z^{2} e^{\left(\lambda-\frac{\rho}{2}\right) z} d z\right] \mu(u) d u d s<0
$$

As $\varphi(\lambda)=\varphi(\rho-\lambda)$, the roots of $\varphi$ are also symmetric according to the line $\operatorname{Re}(\lambda)=\frac{\rho}{2}$. The sign of $\varphi\left(\frac{\rho}{2}\right)=2+B \int_{\tau_{1}}^{\tau_{2}} \mu(s) e^{-\frac{\rho}{2} s} \int_{\tau_{1}}^{\tau_{2}}(u-s)^{2} \mu(u) e^{-\frac{\rho}{2} u} d u d s$ depends on $B$. For $B$ small enough it is negative, otherwise it can be positive.

We can thus deduce that, if $\varphi\left(\frac{\rho}{2}\right)<0$ it means that $\varphi(\lambda)<0$ and since $\Delta^{\prime}(\lambda)=\left(\lambda-\frac{\rho}{2}\right) \varphi(\lambda)$, it implies that $\Delta^{\prime}(\lambda)<0$ if $\lambda>\frac{\rho}{2}$ and $\Delta^{\prime}(\lambda)>0$ if $\lambda<\frac{\rho}{2}$. Thus, as $\Delta\left(\frac{\rho}{2}\right)<0$, there are no real roots to the equation $\Delta(\lambda)=0$ when $B<\frac{-2}{\int_{\tau_{1}}^{\tau_{2}} \mu(s) e^{-\frac{\rho}{2} s} \int_{\tau_{1}}^{\tau_{2}}(u-s) e^{-\frac{\rho}{2} u} \mu(u) d u d s}$

Otherwise if $\varphi\left(\frac{\rho}{2}\right)>0$, then there exists a unique value $\hat{\lambda}(B)<\frac{\rho}{2}$ such that $\varphi(\hat{\lambda}(B))=0$.

We are now going to prove that $\Delta(\hat{\lambda}(B))$ is increasing in $B$.

Taylor expansion of $\Delta(\widehat{\lambda}(B))$ in the neighborhood of $B$ can be written as

$$
\begin{aligned}
\Delta(\widehat{\lambda}(B+\varepsilon))= & \Delta\left(\widehat{\lambda}(B)+\varepsilon \widehat{\lambda}^{\prime}(B)+\varepsilon^{2} \widehat{\lambda}^{(2)}(B)+o\left(\varepsilon^{2}\right)\right) \\
= & \Delta\left(\widehat{\lambda}(B)+\varepsilon \widehat{\lambda}^{\prime}(B)+\varepsilon^{2} \widehat{\lambda}^{(2)}(B)+o\left(\varepsilon^{2}\right)\right) \\
= & \Delta(\widehat{\lambda}(B))+\varepsilon \widehat{\lambda}^{\prime}(B) \Delta^{\prime}(\widehat{\lambda}(B)) \\
& +\frac{1}{2} \varepsilon^{2}\left(\Delta^{\prime}(\widehat{\lambda}(B)) \widehat{\lambda}^{(2)}(B)+\Delta^{(2)}(\widehat{\lambda}(B))\left(\widehat{\lambda}^{\prime}(B)\right)^{2}\right)+o\left(\varepsilon^{2}\right)
\end{aligned}
$$

According to the definition of $\widehat{\lambda}(B)$,

$$
\Delta(\widehat{\lambda}(B+\varepsilon))-\Delta(\widehat{\lambda}(B))=\frac{1}{2}\left(\Delta^{(2)}(\widehat{\lambda}(B))\left(\widehat{\lambda}^{\prime}(B)\right)^{2}\right)+o\left(\varepsilon^{2}\right)<0
$$

We have already seen that for $B$ small enough, $\Delta(\widehat{\lambda}(B))<0$, since for every value of $\lambda, \Delta(\widehat{\lambda}(B))<0$.

Moreover, since we have $\Delta(\widehat{\lambda}(B))$ increasing in $B$ and $\Delta(\widehat{\lambda}(B))$ very high when $B$ approaches 0 , we can conclude that there exists a unique value $B^{D}$ of $B$ such that $\Delta\left(\hat{\lambda}\left(B^{D}\right)\right)=\Delta^{\prime}\left(\hat{\lambda}\left(B^{D}\right)\right)=0$.

For $B$ inferior to $B^{D}$ or superior to $B^{D}$, we thus have respectively 0 or 2 real roots with real parts smaller than $\frac{\rho}{2}$ which means 0 or 4 real roots due to the symmetry. 


\section{B.4 Proof of Lemma 4}

In order to apply the Hopf bifurcation theorem we need to prove that under the conditions given, there exists at least one value of a critical parameter $B^{H}$ such that for $B=B^{H}$ the characteristic equation admits at least one pair of purely imaginary root and then to show that these roots are simple roots and that the crossing velocity of the imaginary axis in the neighborhood of the imaginary solution does not vanish. $B^{H}$ will be such that $B^{H}=\min \left[B^{H 1}, B^{H 2}, B^{H 3}\right]$.

Conditions 3 and 4 will ensure that the existence of pure imaginary roots and the characterization of the corresponding critical parameter. Condition 5 will guarantee that these roots are simple roots.

\section{Existence of imaginary roots and characterization of the critical pa- rameter}

According to expression (17) in the proof B.2, a sufficient condition to have at least one solution to equation $\operatorname{Im}(\Delta(i q))=0$ is that $B<\rho(\varphi(0))^{-1}$, where $\varphi(0)=\int_{\tau_{1}}^{\tau_{2}} \int_{\tau_{1}}^{\tau_{2}}(u-s) e^{-\rho u} \mu(u) d u d s$, which corresponds to Condition 3 .

Let us assume that there exist a pure imaginary root $i q$. According to the previous expression (19), it solves $P(q)=0$, where $P(q)$ has been defined as

$$
P(q)=\left(q^{2}+(\beta+\rho) \beta\right)^{2}+(q \rho)^{2}-B^{2}\left(\begin{array}{c}
{\left[\left(\int_{\tau_{1}}^{\tau_{2}} \mu(s) \cos (q s) d s\right)^{2}+\left(\int_{\tau_{1}}^{\tau_{2}} \mu(s) \sin (q s) d s\right)^{2}\right] *} \\
{\left[\left(\int_{\tau_{1}}^{\tau_{2}} e^{-\rho u} \cos (q u) \mu(u) d u\right)^{2}+\left(\int_{\tau_{1}}^{\tau_{2}} e^{-\rho u} \sin (q u) \mu(u) d u\right)^{2}\right]}
\end{array}\right)
$$

Let us remind that $P$ is an even function, thus we just need to study positive roots $q$.

Since $\lim _{q \rightarrow \pm \infty} P(q)=\infty$, a sufficient condition to have at least one positive real root is $P(0)<0$. Condition 4 is a sufficient condition for $P(0)<0$. If Condition 4 holds, we can thus consider $q_{r}$, one of the roots of $P(q)=0$ and characterize the critical parameter.

Using $\operatorname{Im}\left(\Delta\left(i q_{r}\right)\right)=0$, we can then compute the corresponding critical parameter $B_{r}^{H}$ such that

$$
B_{r}^{H}=\frac{q_{r} \rho}{\int_{\tau_{1}}^{\tau_{2}} \int_{\tau_{1}}^{\tau_{2}} \mu(s) e^{-\rho u} \mu(u) \sin \left(q_{r}(u-s)\right) d u d s}
$$

\section{Simple roots}

We need now to rule out the cases where $q_{r}$ could be a double complex root. If $q_{r}$ were a double root, it would solve $\Delta^{\prime}(i q)=0$, that is to say

$$
\left\{\begin{array}{l}
\operatorname{Re} \Delta^{\prime}(i q)=0 \\
\operatorname{Im} \Delta^{\prime}(i q)=0
\end{array}\right.
$$


with

$$
\begin{aligned}
& \operatorname{Re} \Delta^{\prime}(i q)=-\rho+B \int_{\tau_{1}}^{\tau_{2}} \int_{\tau_{1}}^{\tau_{2}}(u-s) e^{-\rho u} \mu(s) \cos (q(u-s)) \mu(u) d u d s \\
& \operatorname{Im} \Delta^{\prime}(i q)=2 q+B \int_{\tau_{1}}^{\tau_{2}} \int_{\tau_{1}}^{\tau_{2}}(u-s) e^{-\rho u} \mu(s) \sin (q(u-s)) \mu(u) d u d s
\end{aligned}
$$

A necessary condition to have a double root is to have $\operatorname{Im} \Delta^{\prime}(i q)=0$, for $q>0$.

But

$$
\begin{aligned}
\frac{\operatorname{Im} \Delta^{\prime}(i q)}{q} & =2+B \int_{\tau_{1}}^{\tau_{2}} \int_{\tau_{1}}^{\tau_{2}}(u-s)^{2} e^{-\rho u} \mu(s) \frac{\sin (q(u-s))}{q(u-s)} \mu(u) d u d s \\
& <2+B \int_{\tau_{1}}^{\tau_{2}} \int_{\tau_{1}}^{\tau_{2}}(u-s)^{2} e^{-\rho u} \mu(s) \mu(u) d u d s
\end{aligned}
$$

Given the definition of $B$, we have

$$
\frac{\operatorname{Im} \Delta^{\prime}(i q)}{q}<0
$$

Therefore if Condition 5 holds, the roots $q_{r}$ cannot be double roots.

\section{Crossing velocity}

It remains to prove that roots cross the imaginary axis transversally.

Considering $\Delta(\lambda)=0$ as a function of parameter $B$, we can compute the total differentiation of the characteristic equation to get

$$
\Delta^{\prime}(\lambda) d \lambda=-\left(\int_{\tau_{1}}^{\tau_{2}} \mu(s) e^{-\lambda s} \int_{\tau_{1}}^{\tau_{2}} e^{-(\rho-\lambda) u} \mu(u) d u d s\right) d B
$$

thus

$$
\left(\frac{d \lambda}{d B}\right)^{-1}=\frac{2 \lambda-\rho+B \int_{\tau_{1}}^{\tau_{2}} \int_{\tau_{1}}^{\tau_{2}} \mu(s) e^{-\rho u} e^{\lambda(u-s)} \mu(u) d u d s}{-\left(\int_{\tau_{1}}^{\tau_{2}} \mu(s) e^{-\lambda s} \int_{\tau_{1}}^{\tau_{2}} e^{-(\rho-\lambda) u} \mu(u) d u d s\right)}
$$

as $\lambda$ is a root of $\Delta(\lambda)=0$, the previous equation can be rewritten as

$$
\left(\frac{d \lambda}{d B}\right)^{-1}=B \frac{2 \lambda-\rho+B \int_{\tau_{1}}^{\tau_{2}} \int_{\tau_{1}}^{\tau_{2}} \mu(s) e^{-\rho u} e^{\lambda(u-s)} \mu(u) d u d s}{(\lambda+\beta)(\lambda-\rho-\beta)}
$$

Let us now study the sign of $\frac{d \operatorname{Re}(\lambda)}{d B}$. We have

$$
\operatorname{sign}\left(\frac{d \operatorname{Re}(\lambda)}{d B}\right)_{\mid B=B_{r}^{H}}=\operatorname{sign}\left(\operatorname{Re}\left(\frac{d \lambda}{d B}\right)^{-1}\right)_{\mid B=B_{r}^{H}}
$$

Hence, using (B.4,

$$
\operatorname{sign}\left(\frac{d \operatorname{Re}(\lambda)}{d B}\right)_{\mid B=B_{r}^{H}}=\operatorname{sign}(M)
$$


where $M$ is defined as

$$
M=\left(\begin{array}{l}
\left(q_{r}^{2}+\beta(\beta+\rho)\right)\left(-\rho+B_{r}^{H} \int_{\tau_{1}}^{\tau_{2}} \int_{\tau_{1}}^{\tau_{2}} \mu(s) e^{-\rho u}(u-s) \cos \left(q_{r}(u-s)\right) \mu(u) d u d s\right) \\
+2 q_{r} \rho\left(2 q_{r}+B_{r}^{H} \int_{\tau_{1}}^{\tau_{2}} \int_{\tau_{1}}^{\tau_{2}} \mu(s) e^{-\rho u}(u-s) \sin \left(q_{r}(u-s)\right) \mu(u) d u d s\right)
\end{array}\right)
$$

but

$$
\begin{aligned}
& -\rho+B_{r}^{H} \int_{\tau_{1}}^{\tau_{2}} \int_{\tau_{1}}^{\tau_{2}} \mu(s) e^{-\rho u}(u-s) \cos \left(q_{r}(u-s)\right) \mu(u) d u d s \\
< & -\rho+B_{r}^{H} \int_{\tau_{1}}^{\tau_{2}} \int_{\tau_{1}}^{s} \mu(s) e^{-\rho u}(u-s) \cos \left(q_{r}(u-s)\right) \mu(u) d u d s \\
& +B_{r}^{H} \int_{\tau_{1}}^{\tau_{2}} \int_{s}^{\tau_{2}} \mu(s) e^{-\rho u}(u-s) \cos \left(q_{r}(u-s)\right) \mu(u) d u d s \\
< & -\rho+B_{r}^{H} \int_{\tau_{1}}^{\tau_{2}} \int_{\tau_{1}}^{s} \mu(s) e^{-\rho u}(u-s)\left(1-\frac{1}{2}\left(q_{r}(u-s)\right)^{2}\right) \mu(u) d u d s \\
& +B_{r}^{H} \int_{\tau_{1}}^{\tau_{2}} \int_{s}^{\tau_{2}} \mu(s) e^{-\rho u}(u-s) \mu(u) d u d s \\
< & -\rho+B_{r}^{H} \int_{\tau_{1}}^{\tau_{2}} \int_{\tau_{1}}^{\tau_{2}} \mu(s) e^{-\rho u}(u-s) \mu(u) d u d s \\
& -B_{r}^{H} \frac{1}{2} \int_{\tau_{1}}^{\tau_{2}} \int_{\tau_{1}}^{s} \mu(s) e^{-\rho u}(u-s) q_{r}(u-s)^{2} \mu(u) d u d s
\end{aligned}
$$

$\operatorname{As} \int_{\tau_{1}}^{\tau_{2}} \int_{\tau_{1}}^{\tau_{2}} \mu(s) e^{-\rho u}(u-s) \mu(u) d u d s=\varphi(0)<0$ and $\int_{\tau_{1}}^{\tau_{2}} \int_{\tau_{1}}^{s} \mu(s) e^{-\rho u}(u-s) q_{r}(u-s)^{2} \mu(u) d u d s<$ 0 , then

$$
-\rho+B_{r}^{H} \int_{\tau_{1}}^{\tau_{2}} \int_{\tau_{1}}^{\tau_{2}} \mu(s) e^{-\rho u}(u-s) \cos \left(q_{r}(u-s)\right) \mu(u) d u d s<0
$$

Moreover, according to Condition 5 .

$$
2+B_{r}^{H} \int_{\tau_{1}}^{\tau_{2}} \int_{\tau_{1}}^{\tau_{2}} \mu(s) e^{-\rho u}(u-s) \frac{\sin \left(q_{r}(u-s)\right)}{q_{r}} \mu(u) d u d s<0
$$

thus

$$
\operatorname{sign}\left(\frac{d \operatorname{Re}(\lambda)}{d B}\right)_{\mid B=B_{r}^{H}}<0
$$




\title{
Cahiers du GREThA Working papers of GREThA
}

\author{
GREThA UMR CNRS 5113 \\ Université Montesquieu Bordeaux IV \\ Avenue Léon Duguit \\ 33608 PESSAC - FRANCE \\ Tel : +33 (0)5.56.84.25.75 \\ Fax : +33 (0)5.56.84.86.47 \\ http://gretha.u-bordeaux4.fr/
}

\begin{abstract}
Cahiers du GREThA (derniers numéros - last issues)
2012-20 : SALLE Isabelle, ZUMPE Martin, YILDIZOGLU Murat, SENEGAS Marc-Alexandre, Modelling Social Learning in an Agent-Based New Keynesian Macroeconomic Model

2012-21: ABDELLAH Kamel, NICET-CHENAF Dalila, ROUGIER Eric, FDI and macroeconomic volatility: A close-up on the source countries

2012-22 : BECUWE Stéphane, BLANCHETON Bertrand, CHARLES Léo, The decline of French trade power during the first globalization (1850-1913)

2012-23 : ROUILLON Sébastien, An Economic Mechanism to Regulate Multispecies Fisheries

2012-24 : LISSONI Francesco, MONTOBBIO Fabio, The ownership of academic patents and their impact. Evidence from five European countries

2012-25 : PETIT Emmanuel, TCHERKASSOF Anna, GASSMANN Xavier, Sincere Giving and Shame in a Dictator Game

2012-26 : LISSONI Francesco, PEZZONI Michele, POTI Bianca, ROMAGNOSI Sandra, University autonomy, IP legislation and academic patenting: Italy, 1996-2007

2012-27 : OUEDRAOGO Boukary, Population et environnement : Cas de la pression anthropique sur la forêt périurbaine de Gonsé au Burkina Faso.

2012-28 : OUEGRAOGO Boukary, FERRARI Sylvie, Incidence of forest income in reducing poverty and inequalities: Evidence from forest dependant households in managed forest' areas in Burkina Faso

2012-29 : PEZZONI Michele, LISSONI Francesco, TARASCONI Gianluca, How To Kill Inventors: Testing The Massacrator ${ }^{\odot}$ Algorithm For Inventor Disambiguation

2013-01 : KAMMOUN Olfa, RAHMOUNI Mohieddine, Intellectual Property Rights, Appropriation Instruments and Innovation Activities: Evidence from Tunisian Firms

2013-02 : BLANCHETON Bertrand, NENOVSKY Nikolay, Protectionism and Protectionists Theories at the Balkans in the Interwar Period

2013-03 : AUGERAUD-VERON Emmanuelle, LEANDRI Marc, Optimal pollution control with distributed delays
\end{abstract}

La coordination scientifique des Cahiers du GREThA est assurée par Sylvie FERRARI et Vincent FRIGANT. La mise en page est assurée par Anne-Laure MERLETTE. 